\title{
A Multicarrier DS-CDMA System Using Both Time-Domain and Frequency-Domain Spreading
}

\author{
Lie-Liang Yang, Wei Hua and Lajos Hanzo \\ Department of Electronics and Computer Science \\ University of Southampton, SO17 1BJ, UK. \\ Tel: +44-23-8059 3125, Fax: +44-23-8059 4508 \\ Email: 1ly,lh@ecs.soton.ac.uk; http://www-mobile.ecs.soton.ac.uk
}

\begin{abstract}
In this contribution we propose and study a multicarrier direct-sequence code-division multiple-access (MC DSCDMA) scheme, which employs both time (T)-domain and frequency (F)-domain spreading. We investigate the achievable detection performance in the context of synchronous TF-domain spread MC DS-CDMA, when communicating over an additive white Gaussian noise (AWGN) channel. The performance of three different detection schemes is investigated, which include the single-user correlation based detector, the joint TF-domain decorrelating multiuser detector (MUD) and the separate TFdomain decorrelating/MMSE MUD. Our simulation results show that the separate TF-domain decorrelating/MMSE MUD scheme is capable of achieving a better bit error rate (BER) performance than that of the significantly more complex joint TF-domain MUD schemes.
\end{abstract}

\section{INTRODUCTION}

Recently, multicarrier DS-CDMA (MC DS-CDMA) has attracted wide attantion in the field of wireless communications [1], [2]. In MC DS-CDMA each subcarrier signal constitutes a Time (T)-domain DS spread signal, but no frequency $(\mathrm{F})$-domain spreading is employed. In this contribution we propose and study a spread-spectrum scheme, which constitutes an amalgam of the above spread-spectrum schemes. More explicitly, this novel spread-spectrum scheme spreads the transmitted data stream using two signature codes, where one of the signature codes corresponds to the $\mathrm{T}$ domain spreading, while the other corresponds to the F-domain spreading. Since the proposed multicarrier DS-CDMA scheme employs both the above-mentioned T-domain spreading and Fdomain spreading, it is referred to as TF-domain spread MC DS-CDMA.

The benefits of employing both T-domain spreading and Fdomain spreading in MC DS-CDMA systems are multi-fold. Firstly, the future generations of broadband multiple-access systems [3] are expected to have a bandwidth on the order of tens or even hundreds of $\mathrm{MHz}$. When single-carrier based DS-CDMA or MC-CDMA using solely T-domain spreading or solely F-domain spreading is utilized, these broadband systems may inevitably require a high chip-rate and long spreading codes. In the proposed TF-domain spread MC DS-CDMA scheme, the total system bandwidth is related to the product of the T-domain spreading factor and the F-domain spreading factor. Therefore, a relatively low-chip-rate and short spreading codes can be employed in TF-domain spread MC DS-CDMA schemes. Secondly, broadband multiple-access systems are expected to aim for supporting a wide range of services and bit rates, as well as supporting a high number of simultaneous users. When single-carrier DS-CDMA or a MC-CDMA is invoked for the sake of supporting a high number of users, the employment of advanced multiuser detection algorithms becomes impractical owing to their high complexity. By contrast, in the proposed TF-domain spread MC DS-CDMA schemes simultaneous users can be separated in both the Tdomain and the F-domain with the aid of unique signature codes. Furthermore, we will show that multiuser detection can be carried out separately in the T-domain and F-domain, while achieving a similar detection performance to that of joint TFdomain processing. Consequently, the detection complexity of the proposed scheme can be significantly decreased in comparison to that of a conventional single-carrier DS-CDMA or MC-CDMA scheme.

In this contribution we investigate the detection performance of various detection schemes suitable for demodulating the TFdomain spread MC DS-CDMA signals, when communicating over AWGN channels. Specifically, three types of detectors are investigated, which are the single-user correlation based detector, the joint TF-domain decorrelating MUD and the separate TF-domain decorrelating/MMSE MUD [4].

\section{MC DS-CDMA SignALS USING TF-DOMAIN SPREADING}

The transmitter schematic of the TF-domain spread MC DSCDMA system is shown in Fig. 1 in the context of the $k$ th user. At the transmitter side, the binary data stream $b_{k}(t)$ is first DS spread using the T-domain signature sequence $a_{k}(t)$. Following T-domain DS spreading, the spread signal is divided into $M$ parallel branches, where each branch of the signal is multiplied by the corresponding chip value of the F-domain spreading sequence $\mathbf{c}_{k}=\left[c_{k}[1], c_{k}[2], \ldots, c_{k}[M]\right]^{T}$ of length $M$. Following F-domain spreading, each of the $M$ branch signals modulates one of the $M$ subcarrier frequencies using binary phase shift keying (BPSK). Hence, the transmitted signal of user $k$ can be expressed as

$$
s_{k}(t)=\sqrt{\frac{2 P}{M}} \sum_{m=1}^{M} b_{k}(t) a_{k}(t) c_{k}[m] \cos \left(\omega_{m} t\right),
$$




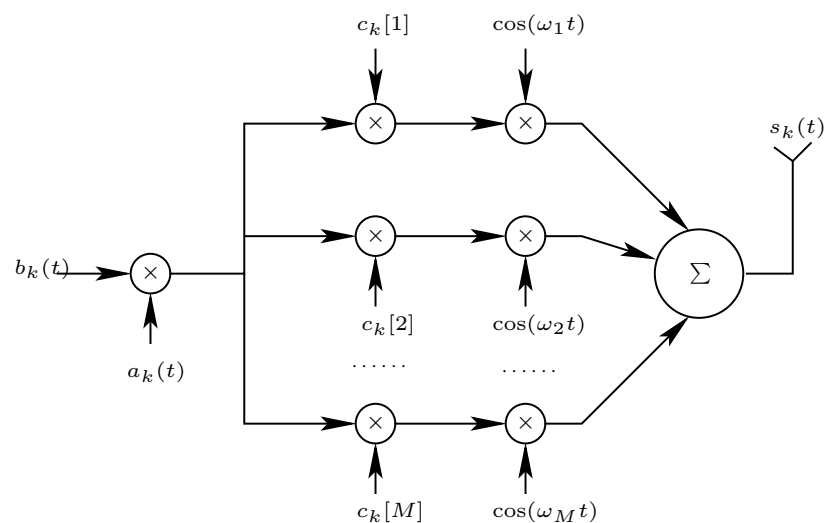

Fig. 1. Transmitter model of MC DS-CDMA using both time-domain and frequency-domain spreading.

where $P$ represents the identical transmitted power of each user, $\left\{\omega_{m}\right\}_{m=1}^{M}$ represents the subcarrier frequency set. The binary data stream's waveform $b_{k}(t)=\sum_{i=0}^{\infty} b_{k} P_{T_{b}}\left(t-i T_{b}\right)$ consists of a sequence of mutually independent rectangular pulses of duration $T_{b}$ and of amplitude +1 or -1 , both having an equal probability. In the T-domain spreading sequence $a_{k}(t)=$ $\sum_{j=0}^{\infty} a_{k j} P_{T_{c}}\left(t-j T_{c}\right)$ of the $k$ th user, $P_{T_{c}}(t)$ represents the rectangular T-domain chip waveform, which is defined over the interval $\left[0, T_{c}\right)$. We assume that the T-domain spreading factor is $N=T_{b} / T_{c}$, which represents the number of chips per bit-duration, and short T-domain spreading sequences are used. Furthermore, we assume that the subcarrier signals are orthogonal and that the spectral main-lobes of the subcarrier signals are not overlapping with each other.

In the system studied $K$ synchronous TF-domain spread MC DS-CDMA signals obeying the form of (1) are transmitted over AWGN channels. We assume that the power received from each user is identical, implying perfect power control. Consequently, the received signal can be expressed as

$r(t)=\sum_{k=1}^{K} \sqrt{\frac{2 P}{M}} \sum_{m=1}^{M} b_{k}(t) a_{k}(t) c_{k}[m] \cos \left(\omega_{m} t\right)+n(t)$,

where $n(t)$ represents the AWGN having zero mean and double-sided power spectral density of $N_{0} / 2$.

As shown in Fig.1 and (1), each TF-domain spread MC DSCDMA signal is spread by two signature sequences, one in the context of the T-domain and another one in the F-domain. The employment of concatenated TF-domain spreading is beneficial in the context of various detection schemes, allowing us to achieve a satisfactory trade-off between the affordable detection complexity and the achievable bit error rate (BER) performance. Let us first consider the single-user detection of TF-domain spread MC DS-CDMA signals.

\section{Single-User Detector}

Let the $u$ th user be the user-of-interest and consider the correlator-based receiver of Fig.2, which carries out the inverse operations of the functions seen in Fig.1. As shown in Fig.2,

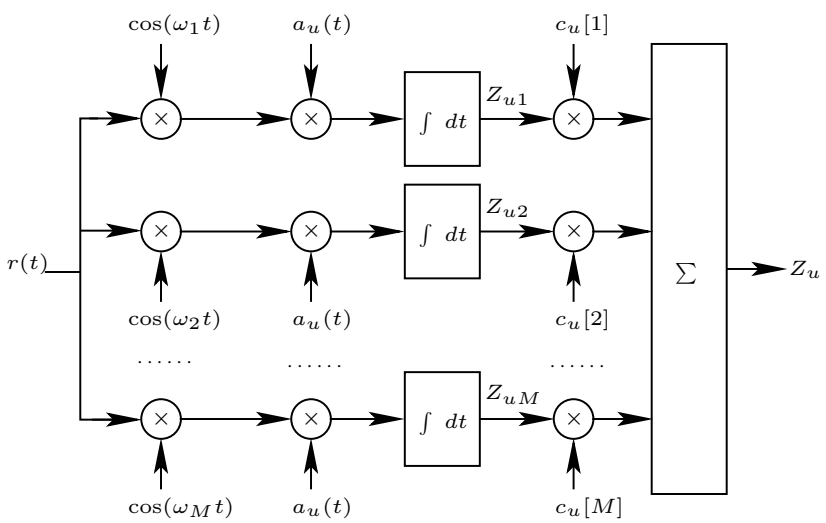

Fig. 2. Receiver model of MC DS-CDMA using both time-domain and frequency-domain spreading.

the output variable related to the first data bit corresponding to the $m$ th subcarrier of the $u$ th user can be expressed as

$$
Z_{u m}=\int_{0}^{T_{b}} r(t) a_{u}(t) \cos \left(\omega_{m} t\right) d t,
$$

where $u=1,2, \ldots, K, m=1,2, \ldots, M$. Upon substituting (2) into (3) and considering the orthogonality between different subcarriers, it can be shown that the output variable $Z_{u m}$ of Fig. 2 can be expressed as

$$
Z_{u m}=\sqrt{\frac{P}{2 M}} T_{b}\left\{\begin{array}{c}
b_{u} c_{u}[m]+\sum_{\substack{k=1 \\
k \neq u}}^{K} b_{k} c_{k}[m] \rho_{u k}+N_{u m} \\
u=1,2, \ldots, K ; m=1,2, \ldots, M .
\end{array}\right.
$$

where $N_{u m}$ is a Gaussian random variable having zero mean and a variance of $M N_{0} / 2 E_{b}$, where $E_{b}=P T_{b}$ represents the energy per bit, while $\rho_{k u}=\frac{1}{T_{b}} \int_{0}^{T_{b}} a_{u}(t) a_{k}(t) d t$ represents the correlation factor between the T-domain spreading sequences $a_{u}(t)$ and $a_{k}(t)$ of users $u$ and $k$.

The decision variable $Z_{u}$ of Fig.2, which corresponds to the first transmitted data bit of the reference user $u$ is obtained by despreading each of the $M$ branch outputs $\left\{Z_{u 1}, Z_{u 2}, \ldots, Z_{u M}\right\}$ using the $u$ th user's F-domain spreading sequence $\mathbf{c}_{u}$, which can be expressed as

$$
\begin{aligned}
Z_{u} & =\sum_{m=1}^{M} c_{u}[m] Z_{u m} \\
& =\sqrt{\frac{P M}{2}} T_{b}\left\{b_{u}+\sum_{\substack{k=1 \\
k \neq u}}^{K} b_{k} \rho_{u k} \beta_{u k}+N_{u}\right\},
\end{aligned}
$$

where $u=1,2, \ldots, K, N_{u}=\frac{1}{M} \sum_{m=1}^{M} c_{u}[m] N_{u m}$, which is a Gaussian random variable having zero mean and a variance of $N_{0} / 2 E_{b}$. Furthermore, in (5) $\beta_{u k}=\frac{1}{M} \sum_{m=1}^{M} c_{u}[m] c_{k}[m]$, which is the correlation factor between the F-domain spreading sequences $\mathbf{c}_{u}$ and $\mathbf{c}_{k}$ assigned to users $u$ and $k$. 
Finally, the data bit $b_{u}$ transmitted by the $u$ th user is decideded as $b_{u}=\operatorname{sgn}\left(Z_{u}\right)$, where $\operatorname{sgn}(\cdot)$ represents a sign function.

\section{Multiuser Detector}

In this section we consider the multiuser detection of TFdomain spread MC DS-CDMA signals. Specifically, both joint and separate TF-domain detection are investigated. The decision statistics of joint TF-domain detection are obtained after both T-domain and F-domain despreading. By contrast, in the context of separate TF-domain detection the multiuser TFdomain spread MC DS-CDMA signals are detected in a first step in the T-domain, followed by detection in the F-domain.

\section{A. Joint TF-domain Decorrelating MUD}

By observing (5) and considering the detection of $K$ users, it can be shown that after removing the common factor of $\sqrt{\frac{P M}{2}} T_{b}$ associated with all the different users, the decision variables of the $K$ users can be written as

$$
\mathbf{Z}=\mathbf{R b}+\mathbf{n},
$$

where we have

$$
\begin{aligned}
& \mathbf{Z}=\left[Z_{1}, Z_{2}, \ldots, Z_{K}\right]^{T}, \\
& \mathbf{b}=\left[b_{1}, b_{2}, \ldots, b_{K}\right]^{T}, \\
& \mathbf{n}=\left[N_{1}, N_{2}, \ldots, N_{K}\right]^{T}
\end{aligned}
$$

and

$$
\mathbf{R}=\left(\begin{array}{cccc}
1 & \rho_{12} \beta_{12} & \ldots & \rho_{1 K} \beta_{1 K} \\
\rho_{21} \beta_{21} & 1 & \ldots & \rho_{2 K} \beta_{2 K} \\
\vdots & \vdots & \ddots & \vdots \\
\rho_{K 1} \beta_{K 1} & \rho_{K 2} \beta_{K 2} & \ldots & 1
\end{array}\right)
$$

Furthermore, in (6) $\mathbf{n}$ is a zero-mean random Gaussian vector having a covariance of

$$
E\left[\mathbf{n n}^{T}\right]=\frac{N_{0}}{2 E_{b}} \mathbf{R} .
$$

In the context of the joint TF-domain decorrelating MUD the final decision variables associated with $b_{k}, k=$ $1,2, \ldots, K$ can be expressed as

$$
\mathbf{R}^{-1} \mathbf{Z}=\mathbf{b}+\mathbf{R}^{-1} \mathbf{n},
$$

and the corresponding data bit $b_{k}$ is classified according to $\hat{b}_{k}=\operatorname{sgn}\left(\left(\mathbf{R}^{-1} \mathbf{Z}\right)_{k}\right)$ for $k=1,2, \ldots, K$.

\section{B. Separate TF-domain Decorrelating/MMSE MUD}

Let $\left\{a_{1}(t), a_{2}(t), \ldots, a_{N}(t)\right\}$ and $\left\{\mathbf{c}_{1}, \mathbf{c}_{2}, \ldots, \mathbf{c}_{M}\right\}$ be the $N$ number of T-domain spreading sequences and $M$ number of F-domain spreading sequences, respectively, where $\mathbf{c}_{u}=\left[c_{u}[1] \quad c_{u}[2] \ldots c_{u}[M]\right]^{T}, u=1, \ldots, M$ represents a F-domain spreading code. Furthermore, we assume that the number of active users is $K$ and we introduce a new variable of $\mathcal{K}=\lfloor K / N\rfloor$, where $\lfloor x\rfloor$ represents the smallest integer not less than $x$. Then, we have $1 \leq \mathcal{K} \leq M$, since $K \leq N M$. With the above assumptions, the $K$ number of users supported can be grouped into $N$ user groups, with each group supporting at most $\mathcal{K}$ users. Consequently, it can be readily shown that each of the $N$ user groups can be distinguished by assigning one of the $N$ number of T-domain spreading sequences $\left\{a_{1}(t), a_{2}(t), \ldots, a_{N}(t)\right\}$. By contrast, the $1 \leq \mathcal{K} \leq M$ number of user signals of a given group are distinguishable with the aid of the $\mathcal{K}$ number of $\mathrm{F}$-domain spreading sequences chosen from the set $\left\{\mathbf{c}_{1}, \mathbf{c}_{2}, \ldots, \mathbf{c}_{M}\right\}$. Specifically, for the users belonging to the $n$th group the transmitted signals can be expressed as

$$
\begin{array}{r}
s_{n}(t)=\sqrt{\frac{2 P}{M}} \sum_{\kappa=1}^{\mathcal{K}} \sum_{m=1}^{M} b_{n \kappa}(t) a_{n}(t) c_{\kappa}[m] \cos \left(\omega_{m} t\right), \\
n=1,2, \ldots, N
\end{array}
$$

where $b_{n \kappa}(t)$ is the transmitted waveform of the user corresponding to the $n$th group, $n=1,2, \ldots, N$ - which is distinguished by the T-domain spreading sequence $a_{n}(t)$ using the $\kappa$ th F-domain spreading code for $\kappa=1,2, \ldots, \mathcal{K}$.

The received signal is the composite multiuser signal constituted by the superposition of the signals of the $N$ user groups plus the AWGN contribution of the channel, which can be expressed as

$$
\begin{array}{r}
r(t)=\sqrt{\frac{2 P}{M}} \sum_{n=1}^{N} \sum_{\kappa=1}^{\mathcal{K}} \sum_{m=1}^{M} b_{n \kappa}(t) a_{n}(t) c_{\kappa}[m] \cos \left(\omega_{m} t\right) \\
+n(t) .
\end{array}
$$

The transmitted data of a given user can be detected with the aid of its T-domain group signature sequence as well as its F-domain spreading sequence - which can also be described as its user signature sequence. Let $Z_{u}[m], u=$ $1,2, \ldots, N$, represent the receiver's output with respect to the $m$ th subcarrier in the $u$ th user group, after despreading using the $u$ th group's T-domain signature sequence $a_{u}(t)$. Then, the outputs associated with the $N$ groups can be expressed as

$$
\mathbf{Z}[m]=\mathbf{R}_{t} \mathbf{b}_{g}+\mathbf{n}_{m}, m=1,2, \ldots, M
$$

where the subscript $g$ in $\mathbf{b}_{g}$ is used for emphasising that the current detection stage is at the group level, while the subscript $t$ associated with $\mathbf{R}_{t}$ emphasizes that it is the cross-correlation matrix of the T-domain spreading sequences. Furthermore, the terms in (15) are detailed as follows:

$$
\begin{gathered}
\mathbf{Z}[m]=\left[Z_{1}[m], Z_{2}[m], \ldots, Z_{N}[m]\right]^{T} \\
\mathbf{b}_{g}=\left[\sum_{\kappa=1}^{\mathcal{K}} b_{1 \kappa} c_{\kappa}[m], \sum_{\kappa=1}^{\mathcal{K}} b_{2 \kappa} c_{\kappa}[m], \ldots, \sum_{\kappa=1}^{\mathcal{K}} b_{N \kappa} c_{\kappa}[m]\right]^{T} \\
\mathbf{n}_{m}=\left[N_{1 m}, N_{2 m}, \ldots, N_{N m}\right]^{T} \\
\mathbf{R}_{t}=\left(\begin{array}{cccc}
1 & \rho_{12} & \ldots & \rho_{1 N} \\
\rho_{21} & 1 & \ldots & \rho_{2 N} \\
\vdots & \vdots & \ddots & \vdots \\
\rho_{N 1} & \rho_{N 2} & \ldots & 1
\end{array}\right)
\end{gathered}
$$


Note that, $\mathbf{n}_{m}$ of (18) is a zero-mean Gaussian random vector having a covariance matrix of

$$
E\left[\mathbf{n}_{m} \mathbf{n}_{m}^{T}\right]=\frac{M N_{0}}{2 E_{b}} \mathbf{R}_{t} .
$$

For the separate TF-domain decorrelating/MMSE MUD, the $N$ variables of (15) associated with the $N$ user groups are first processed by a decorrelator with respect to each of the $M$ subcarriers and the results can be expressed as

$$
\mathbf{z}_{m}=\mathbf{R}_{t}^{-1} \mathbf{Z}[m]=\mathbf{b}_{g}+\mathbf{R}_{t}^{-1} \mathbf{n}_{m}, m=1,2, \ldots, M,
$$

where $\mathbf{z}_{m}$ is an $N$-dimensional vector, representing the soft outputs of the group-signal decorrelators corresponding to the $N$ number of groups after the decorrelation process, and $\mathbf{R}_{t}^{-1} \mathbf{n}_{m}$ is a random Gaussian vector having the covariance matrix given by

$$
E\left[\left(\mathbf{R}_{t}^{-1} \mathbf{n}_{m}\right)\left(\mathbf{R}_{t}^{-1} \mathbf{n}_{m}\right)^{T}\right]=\frac{M N_{0}}{2 E_{b}} \mathbf{R}_{t}^{-1}
$$

After the decorrelation process, the composite multiuser signal contained in the $n$th user group is constituted by the $n$th element of $\mathbf{z}_{m}$, which is represented as $\left(\mathbf{z}_{m}\right)_{n}$ and can be expressed as

$$
\begin{gathered}
\left(\mathbf{z}_{m}\right)_{n}=\sum_{\kappa=1}^{\mathcal{K}} b_{n \kappa} c_{\kappa}[m]+\left(\mathbf{R}_{t}^{-1} \mathbf{n}_{m}\right)_{n}, \\
n=1,2, \ldots, N ; m=1,2, \ldots, M .
\end{gathered}
$$

With the aid of (23), the $\mathcal{K}$ number of users contained in the $n$th group can now be uniquely identified by their corresponding F-domain spreading sequences $\left\{\mathbf{c}_{1}, \mathbf{c}_{2}, \ldots, \mathbf{c}_{\mathcal{K}}\right\}$. After the F-domain despreading, we obtain

$$
\mathbf{F}_{n}=\mathbf{R}_{f} \mathbf{b}_{n}+\mathbf{n}_{f}
$$

where we have

$$
\begin{aligned}
\mathbf{F}_{n}= & {\left[F_{n 1}, F_{n 2}, \ldots, F_{n \mathcal{K}}\right]^{T} } \\
\mathbf{b}_{n}= & {\left[b_{n 1}, b_{n 2}, \ldots, b_{n \mathcal{K}}\right]^{T}, } \\
\mathbf{R}_{f}= & \left(\begin{array}{cccc}
1 & \beta_{12} & \ldots & \beta_{1 \mathcal{K}} \\
\beta_{21} & 1 & \ldots & \beta_{2 \mathcal{K}} \\
\vdots & \vdots & \ddots & \vdots \\
\beta_{\mathcal{K} 1} & \beta_{\mathcal{K} 2} & \ldots & 1
\end{array}\right)
\end{aligned}
$$

where $\beta_{i j}$ is the correlation factor of the F-domain spreading sequences $\mathbf{c}_{i}$ and $\mathbf{c}_{j}$, as defined previously in Section III. Furthermore, in (24) the subscript $f$ associated with $\mathbf{R}_{f}$ emphasizes that $\mathbf{R}_{f}$ is the cross-correlation matrix of the Fdomain spreading sequences, while $\mathbf{n}_{f}$ is a $\mathcal{K}$-dimensional random Gaussian vector having a zero-mean and a covariance matrix given by

$$
E\left[\mathbf{n}_{f} \mathbf{n}_{f}^{T}\right]=\frac{N_{0}}{2 E_{b}}\left(\mathbf{R}_{t}^{-1}\right)_{n n} \mathbf{R}_{f} .
$$

Finally, the MMSE solution based F-domain MUD can be implemented as

$$
\begin{aligned}
\left(\mathbf{R}_{f}+\frac{N_{0}}{2 E_{b}}\left(\mathbf{R}_{t}^{-1}\right)_{n n} \mathbf{I}\right)^{-1} \mathbf{F}_{n}= \\
\left(\mathbf{R}_{f}+\frac{N_{0}}{2 E_{b}}\left(\mathbf{R}_{t}^{-1}\right)_{n n} \mathbf{I}\right)^{-1} \mathbf{R}_{f} \mathbf{b}_{n} \\
+\left(\mathbf{R}_{f}+\frac{N_{0}}{2 E_{b}}\left(\mathbf{R}_{t}^{-1}\right)_{n n} \mathbf{I}\right)^{-1} \mathbf{n}_{f}
\end{aligned}
$$

while for each given user group $n$, where $n=1,2, \ldots, N$, the corresponding data bits $b_{n \kappa}$ are decided according to $\hat{b}_{n \kappa}=\operatorname{sgn}\left(\left(\left(\mathbf{R}_{f}+\frac{N_{0}}{2 E_{b}}\left(\mathbf{R}_{t}^{-1}\right)_{n n} \mathbf{I}\right)^{-1} \mathbf{F}_{n}\right)_{\kappa}\right)$ for $\kappa=$ $1,2, \ldots, \mathcal{K}$.

Note that as shown in (12), the joint TF-domain decorrelating detector has to compute the inverse of $\mathbf{R}$, which has the rank determined by the number of users $K$, which may be as high as $M N$. Therefore, the complexity of the joint TF-domain decorrelating detector is on the order of $O\left(K^{3}\right)$, and is of $O\left(M^{3} N^{3}\right)$, respectively, when the system operates under full user load. By contrast, in the context of the separate TF-domain decorrelating/MMSE MUD, the detector is characterized by (21) and (29). The rank of the correlation matrices associated with the T-domain detection is $N$, while the rank of the correlation matrices associated with the Fdomain detection is $\mathcal{K}$, where $\mathcal{K}$ may be as high as $M$, when the system is fully loaded. Hence, in the context of separate TF-domain detection we have to compute the inverse of a $N$-rank matrix for the sake of T-domain detection associated with each of the $M$ subcarriers. Similarly, the inverse of a $\mathcal{K}$-rank matrix associated with each of the $N$ user groups has to be determined for the sake of the corresponding Fdomain detection. Notice that the inverse of the $N$-rank matrix required for T-domain detection only has to be computed once and it can be used for all subcarriers. Similarly, the inverse of the $\mathcal{K}$-rank matrix required for F-domain detection is also computed only once and it can be used for all user groups. Therefore, the complexity of the separate TF-domain detection schemes is on the order of $O\left(N^{3}+\mathcal{K}^{3}\right)$, which is limited to $O\left(N^{3}+M^{3}\right)$, when the system is fully loaded.

\section{Performance Results}

Figs. 3 and 4 compare the BER versus SNR per bit or $E_{b} / N_{0}$ performance of TF-domain spread MC DS-CDMA using the parameters of $N=15, M=7$ for the cases of both light user load in Fig. 3 and for full-load in Fig. 4. The results show that for both types of load the correlation based single-user detector exhibits the worst performance, in particular, when $E_{b} / N_{0}$ is high. Explicitly, the BER curve of the correlation based detector exhibits an error floor at high SNR per bit values. Furthermore, we observe that the separate TF-domain decorrelating/MMSE outperforms the joint TF-domain decorrelating MUD. However, for the light-load scenario, the BER performance of the separate TF-domain decorrelating/MMSE MUD is close to that of the joint TFdomain decorrelating MUD. 


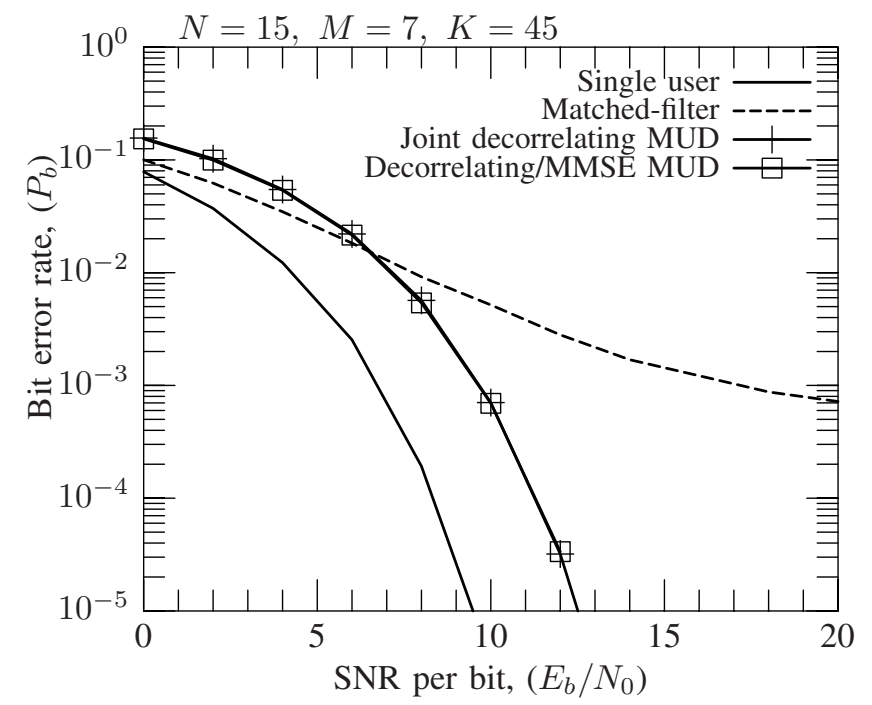

Fig. 3. BER performance comparison of the TF-domain spread MC DSCDMA systems in conjunction with various detection schemes, when using a T-domain spreading factor of $N=15$, F-domain spreading factor of $M=7$ and supporting a total of $K=45$ users.

In Fig.5 we compare the BER versus $E_{b} / N_{0}$ performance of TF-domain spread MC DS-CDMA using the parameters of $N=7, M=15$ for the case of full-load. The results show that all the arguments associated with Fig.4 are applicable also in the context of this figure.

In summary, our study demonstrated that the separate TF-domain decorrelating/MMSE MUD scheme is capable of achieving a better BER performance than that of the joint decorrelating TF-domain MUD schemes, while imposing a significantly lower detection complexity than the joint decorrelating TF-domain MUD schemes.

\section{ACKNOWLEDGMENT}

This work has been funded in the framework of the IST project IST-2001-34091 SCOUT, which is partly funded by the European Union. The authors would like to acknowledge the contributions of their colleagues.

\section{REFERENCES}

[1] R. Prasad and S. Hara, "Overview of multicarrier CDMA," IEEE Communications Magazine, pp. 126-133, December 1997.

[2] L.-L. Yang and L. Hanzo, "Performance of generalized multicarrier DS-CDMA over Nakagami- $m$ fading channels," IEEE Transactions on Communications, vol. 50, pp. 956 - 966, June 2002.

[3] M. Progler, C. Evci, and M. Umehira, "Air interface access schemes for broadband mobile systems," IEEE Communications Magazine, pp. 106115, September 1999.

[4] S. Verdu, Multiuser Detection. Cambridge University Press, 1998.

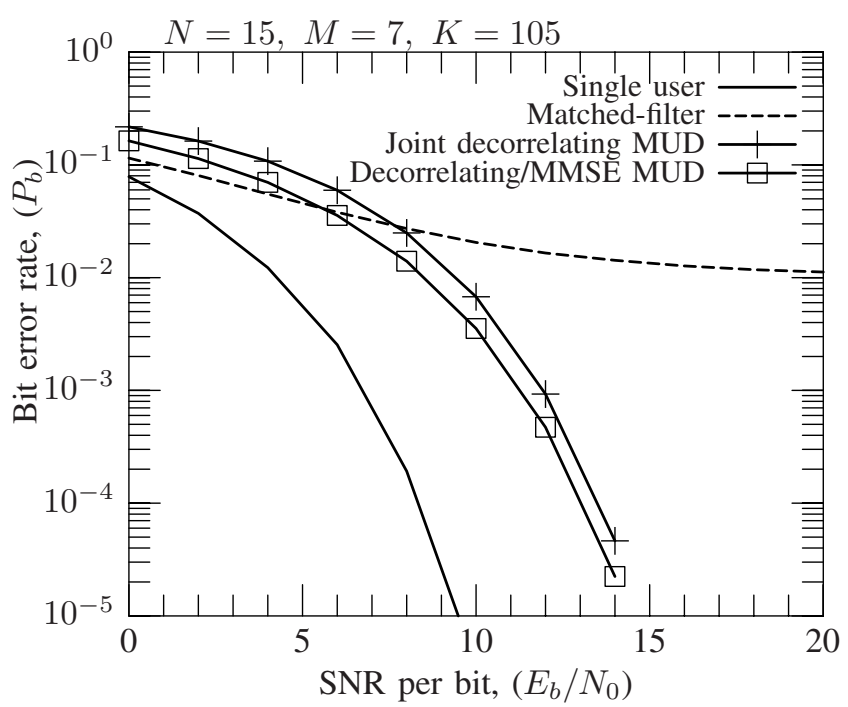

Fig. 4. BER performance comparison of the TF-domain spread MC DSCDMA systems in conjunction with various detection schemes, when using a T-domain spreading factor of $N=15, \mathrm{~F}$-domain spreading factor of $M=7$ and supporting a total of $K=105$ users.

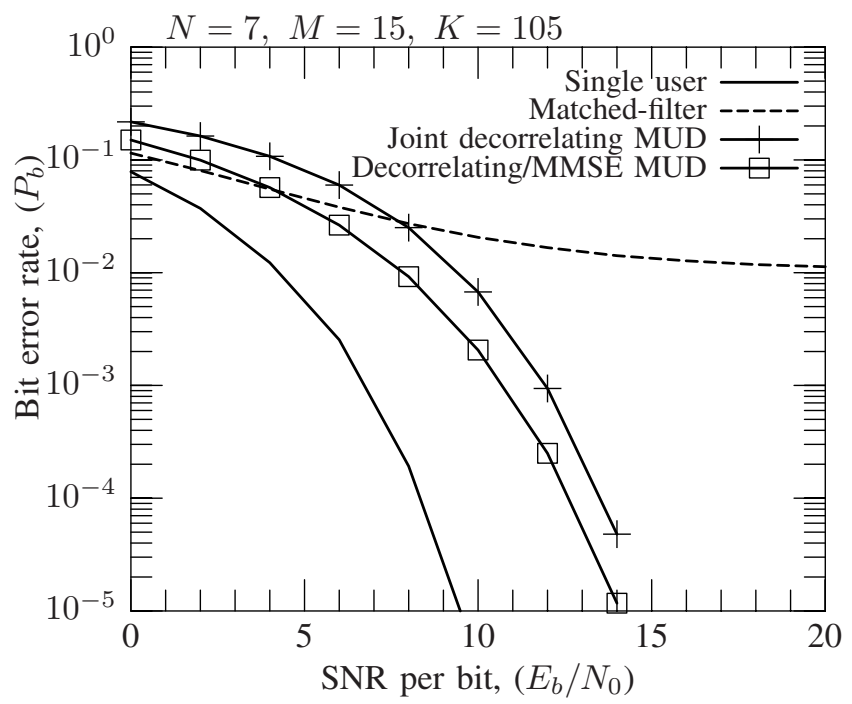

Fig. 5. BER performance comparison of the TF-domain spread MC DSCDMA systems in conjunction with various detection schemes, when using a T-domain spreading factor of $N=7, \mathrm{~F}$-domain spreading factor of $M=15$ and supporting a total of $K=105$ users. 